\title{
Phototactive Thin Films in Science Education
}

\author{
Michael W. Tausch* \\ Bergische Universität Wuppertal, Gaußstr. 20, D-42119 Wuppertal, Germany \\ *Corresponding author: mtausch@uni-wuppertal.de
}

Cite This Article: Michael W. Tausch, "Phototactive Thin Films in Science Education." World Journal of Chemical Education, vol. 6, no. 1 (2018): 14-17. doi: 10.12691/wjce-6-1-3.

\section{Why Photoprocesses in Science Education?}

A fundamental demand on science education today is to communicate core principles of chemistry, physics, biology and informatics in close combination with everyday life experiences of students as well as with convincing applications from modern science and technology. Photochemical and photophysical processes in thin films are par excellence suitable to fulfill this requirement ${ }^{1}$. Therefore research in science education is challenged to develop experiments, concepts and teaching materials which help to interpret and communicate photoprocesses in thin films in a manner, that it is both, exciting and understandable. Adequate teaching concepts, experiments and materials have to bridge the gap between the state of the art in science and technology and the everyday educational activities in high schools, colleges and universities. Guided by A. Einstein's aphorism that " $a$ pretty experiment quite often is more precious than twenty equations bred in the thinking retort", research in chemical education is challenged to investigate and develop experiments strongly focused on the concepts intended to be communicated.

For teaching photoprocesses, N. J. Turro's paradigm of the "excited states of molecules" as "the heart of all photoprocesses" and their interpretation as "an electronic isomer of the ground state" [2], represents a big idea. This core concept has already been used for the experimental approach and the theoretical explanation of different photoprocesses without and with chemical transformation at a level suitable for high schools, colleges and basic courses at universities $[3,4,5,6]$.

According to Turro's statement cited above, the excited state $A^{*}$, the "heart" of all photoprocesses, not even is necessarily generated by light irradiation, it can also emerge from an exergonic chemical reaction or by supplying of electrical energy.

\footnotetext{
${ }^{1}$ Further reasons for the inclusion of photoprocesses into science education given in the talk concern the global challenges in the $21^{\text {th }}$ century (energy, water, food, climate and mobility), as well as the estimated global energy mix until the end of this century. The presentation slights containing these data are provided on reference [1]
}

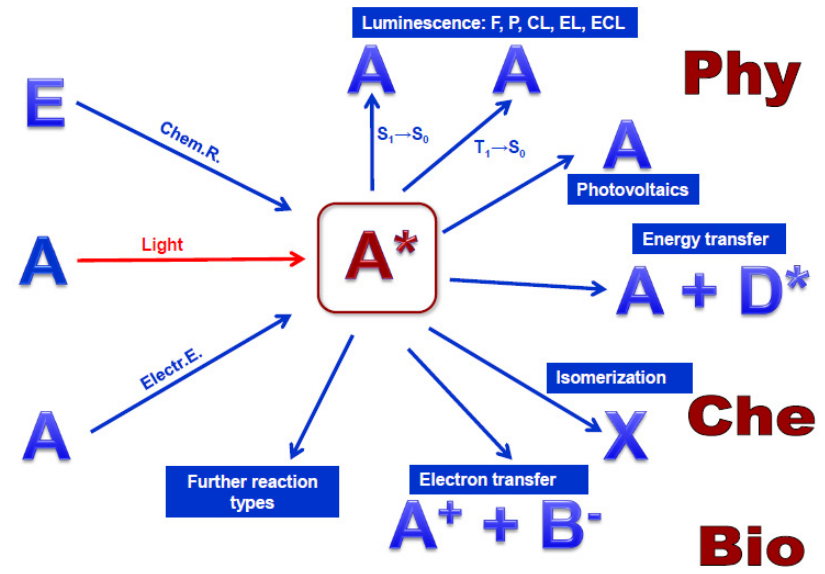

Figure 1. Generation and deactivation paths of the electronically excited state $A^{*}$

Actually, the excited state $A^{*}$ can deactivate or react in very different ways too. So a veritable zoo of photoprocesses can be obtained (see Figure 1). Traditionally some of them are allocated more to physics, others more to biology. However, if one agrees with Turro that $\mathrm{A}^{*}$ is an electronic isomer of $\mathrm{A}$, all photoprocesses indicated in Figure 1 (and others) have to be considered as photochemistry.

\section{Exciting Experiments for the Concept of Electronically Excited States}

Due to their large applications luminescence phenomena, especially fluorescence, phosphorescence and electroluminescence nowadays (in contrast to the past, for instance 30 years ago) count among the everyday experiences of everyone ${ }^{2}$. The phenomena of fluorescence and phosphorescence provide convincing phenomena for an experimental approach to the paradigm of ground state and excited states.

\footnotetext{
${ }^{2}$ Nevertheless, fluorescence and phosphorescence count also among the topics in scientific research at the highest level.

Note that the Nobel Prices in Physics and Chemistry 2014 have been awarded for the blue LEDs and for the STED-Nanoscopy respectively.
} 

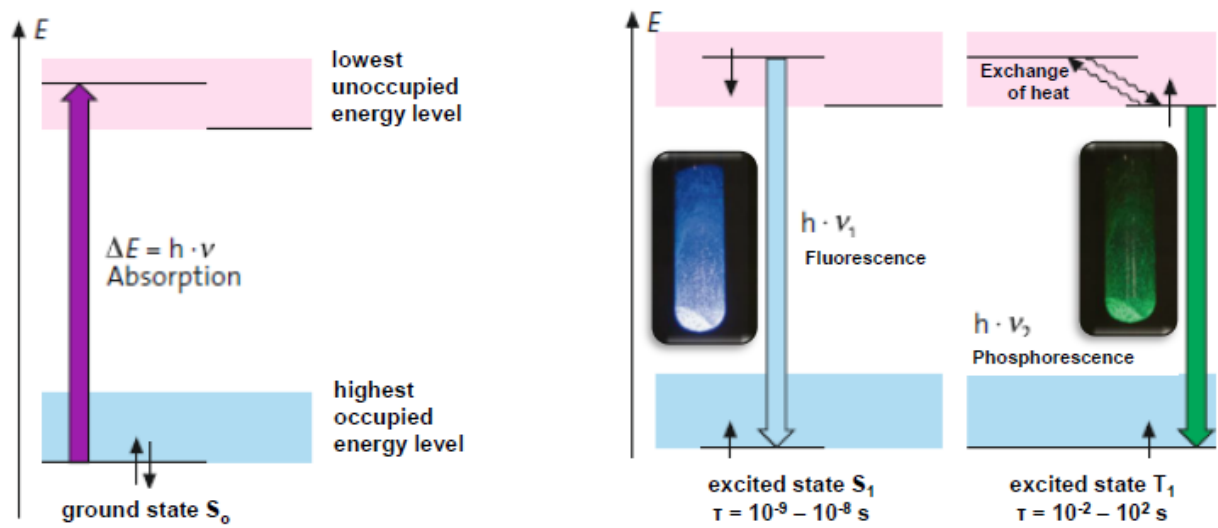

Figure 2. The energy level model - a core concept in chemistry

It would be a "pretty" experiment, if one could succeed in differentiating between fluorescence and phosphorescence as shown in Figure 2 using one and the same probe of a photoluminescent material. The indicated blue fluorescence and the greenish phosphorescence would be in full agreement with the theory that requires that the radiative deactivation $\mathrm{S}_{1}-\mathrm{S}_{0}$, should be more energetic than the radiative deactivation $\mathrm{T}_{1}-\mathrm{S}_{0}$. And the huge difference of the life times of $S_{1}$ and $T_{1}$ should lead to that amazing observation that bluish fluorescence disappears immediately when the exciting UV lamp is switched off, whereas the greenish phosphorescence comes up at the same moment and decays exponentially between a couple of seconds depending on the temperature of the probe.

Actually, such a probe can be prepared in classroom experiments by melting in a test tube approximately $4 \mathrm{~g}$ of tartric acid, adding about $5 \mathrm{mg}$ of aesculine (see Figure 3) and distributing the melted mixture over the inner surface of the test tube by rotating it. After cooling at room temperature the photoactive (more or less thin) layer of aesculine immobilized in tartric acid makes visible even to the naked eye the bluish fluorescence and the greenish phosphorescence as indicated in Figure 3. For explaining this luminescence behavior, especially the red shift of the phosphorescence compared to the fluorescence, supporting teaching materials such as animated energy diagrams from [7] can be used ${ }^{3}$.

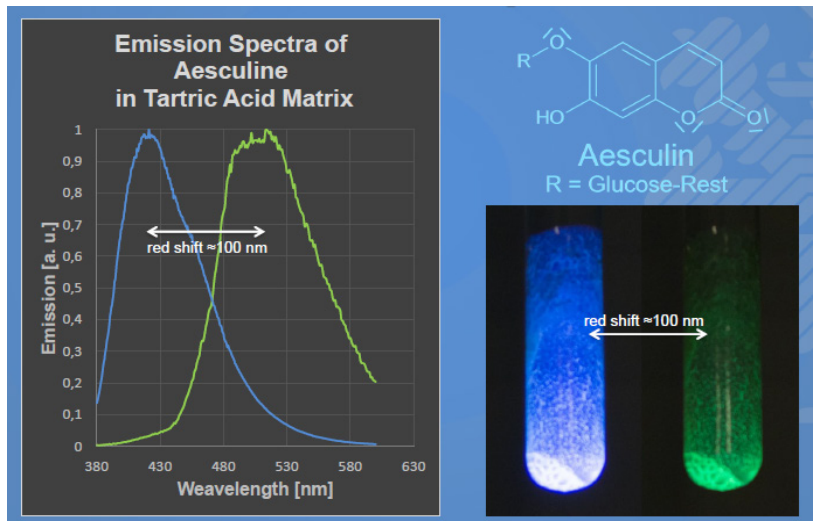

Figure 3. Fluorescence and phosphorescence of aesculine immobilized in tartric acid

\footnotetext{
${ }^{3}$ A kit "Photo-Mol" (Photones and Molecules) containing experimental equipment, work sheets, animations etc. has been developed. It is available from the Hedinger Company in Stuttgart (Germany).
}

It is recommendable to extend the investigation with this probe as following: If the thermal equilibrium between $S_{1}$ and $T_{1}$ (see Figure 2) really occurs with exchange of heat, a longer duration of phosphorescence should be achievable, if the sample would be cooled for trapping the excited $\mathrm{T}_{1}$-state. Otherwise, if the probe would be heated, only a very short or even no phosphorescence should be observed and the color of the fluorescence should turn from bluish to a deep blue. Indeed, these hypotheses can convincingly be confirmed by cooling or heating the probes and observing exactly the color of the fluorescence and the duration of the phosphorescence.

\section{Thin Film Experiments for Core Concepts of Chemistry}

Instant and inexpensive, secure and clean, innovative and "green" experiments can be carried out with an "intelligent" foil accessible by the "Photo-Mol" kit indicated in footnote 3 . The photoactive layer on this foil consists of a thin film containing the molecular switch spiropyrane/merocaynine embedded in polystyrene. The hazard-free and commercially available Spiropyrane (Figure 4) is really a dream compound for teaching photochemistry. In an introductory experiment in toluene solution it can be converted rapidly by simple irradiation with blue light into his isomer merocyanine, and this one comes spontaneously back to spiropyrane in darkness $[7,8]$.

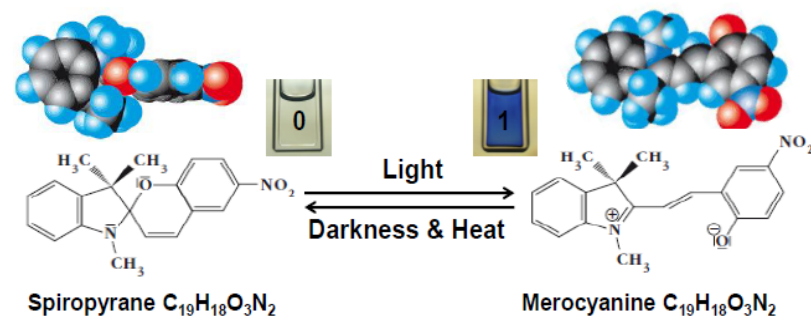

Figure 4. Spiropyrane isomerizes reversibly into merocyanine

Furthermore, this system is suitable for the demonstration and investigation of: i) photoisomerization and molecular switches, ii) the influence of wavelength and of the temperature on a photochemical reaction compared to a thermal reaction, iii) the influence of the nano environment produced by the solvent or the matrix, 
iv) the importance of thin layers for photochemical processes, v) the principle of aggregation induced luminescence, vi) the principle of molecular logics and vii) the concept of photosteady state compared to the thermodynamic equilibrium.

The "intelligent" foil can easily be prepared by dissolving approximately $50 \mathrm{mg}$ of spiropyrane in $15 \mathrm{~mL}$ of toluene, making this solution viscous like honey by addition of polystyrene then brushing this mixture on a PET-foil (see Figure 5).

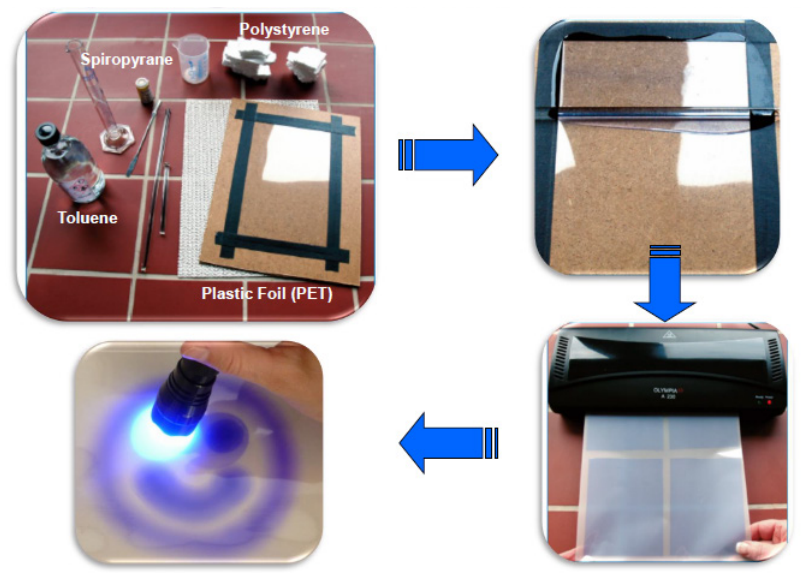

Figure 5. Preparation of an "intelligent" foil (see description in the text)

After the evaporation of the solvent (that takes at room temperature about 20 minutes) the solid photoactive film layer should be protected. For doing so, the whole foil can be packed into an envelope of polyethylene using a laminating machine.

Optical information visible as blue coloration can be written by irradiation with a blue LED torch (or with sunlight using a mask). The information can be erased in a couple seconds by warming up the foil in hot water. Keeping the foil at low temperature (for example in the fridge) in darkness, the information can be stored for hours and days. The blue zones of the foil can be rewritten with new information using a green laser pointer or a green LED torch.

For science education at low level these experiments should be interpreted only from the phenomenological point of view in the sense that chemical reactions can be energetically driven by light or by heat and that light of different colors induce different chemical reactions. Additionally, using stacks of 2-5 "intelligent" foils, the importance of thin films for chemical effects produced by light irradiation can simply and convincingly be demonstrated.

However, for science education at high school and college level these experiments should be used for overcoming gaps in the deeper understanding of theoretical concepts. The main purpose is, to communicate the fundamental difference between a photochemical reaction and a thermal reaction at molecular level. Therefore the concept of ground state (GS) and excited state (ES) can be used again in a modified shape containing the energy curves of the GS and the ES (see Figure 6). The abscissa in this diagram represents the reaction coordinate that concerns changes of nuclear geometry during the reaction. The ordinate represents the energy of the reacting system. The blue curve describes the energetic pathway of a thermal reaction that always occurs exclusively in the GS. It starts from the energetic minimum of a molecule and leads over an energy barrier to the energetic minimum of another molecule. The activation energy indicated in Figure 6 as $E_{a}$ must be supplied as heat. In our case environmental heat at room temperature is sufficient to overcome $\mathrm{E}_{\mathrm{a} 2}$ and to drive the reaction from the blue merocyanine to the yellow spiropyrane. In contrast, the spiropyrane molecules don't react in this way, the molecules would break somewhere in the region before the maximum of the activation energy barrier into fragments instead. Nevertheless, if a spiropyrane molecule is excited by absorption of a bluelight quantum and, if it doesn't fall down immediately emitting a fluorescence quantum, then the excited molecule can "move" along the red curve from Figure 6. This means a consecutive rearrangement of nuclei that is the adoption of new geometrical configurations of atoms in the reacting system - chemically spoken the system undergoes an isomerisation route. The movement along the ES-curve occurs towards the decrease in energy and it leads into an "energy funnel". From the bottom of that funnel the molecule falls down into the GS landing close to the energy maximum of the GS-curve, may be a little on the right side of the maximum. From there it slides to the energetic valley of the product. Students should understand that the energetic pathway of a photochemical reaction always includes the electronically excited state of the reacting system and the corresponding energy profile curve [9].

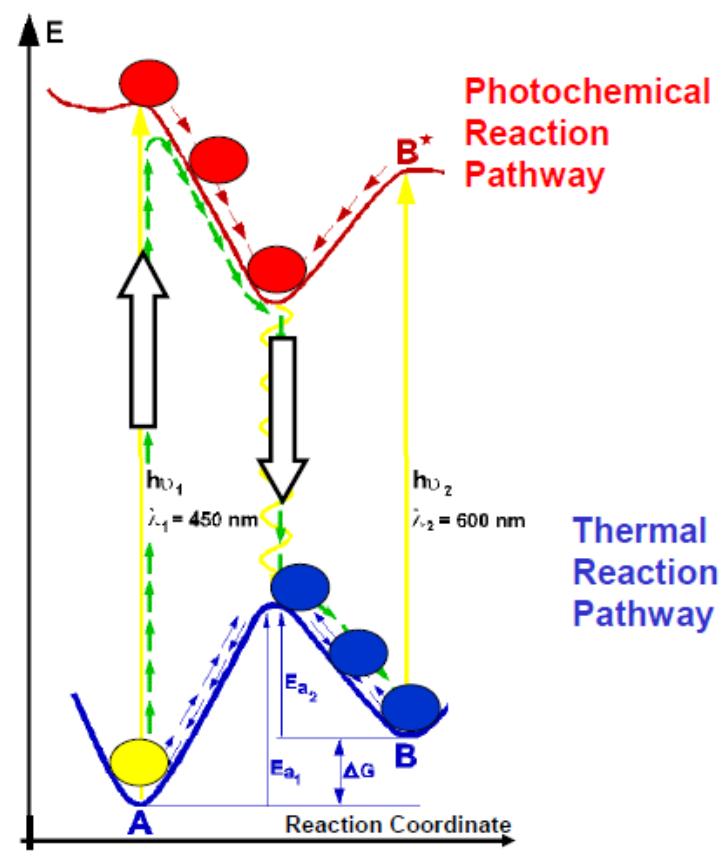

Figure 6. Energy profile curves for a thermal and a photochemical isomerisation

It's aimed to point out, that experimental investigations with the "intelligent" foil at different temperatures and with different wavelengths also offer a good opportunity to mention or even to introduce the concept of photosteady state. It is basically different from the thermodynamic equilibrium. Unlike the thermodynamic equilibrium, the photosteady state is achieved and maintained only by irradiating the reacting system [10]. 


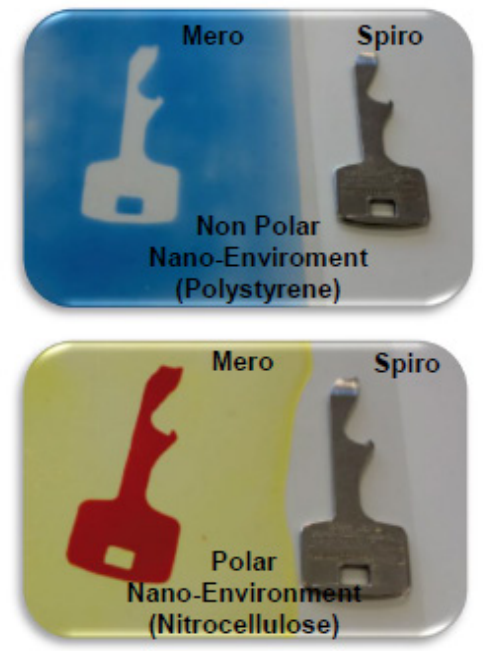

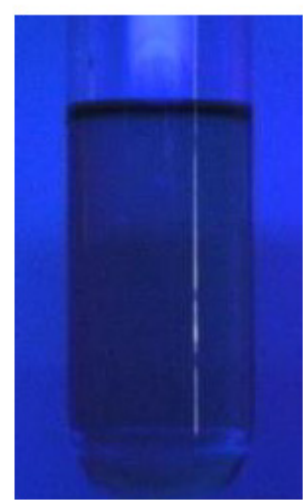

Toluene solution

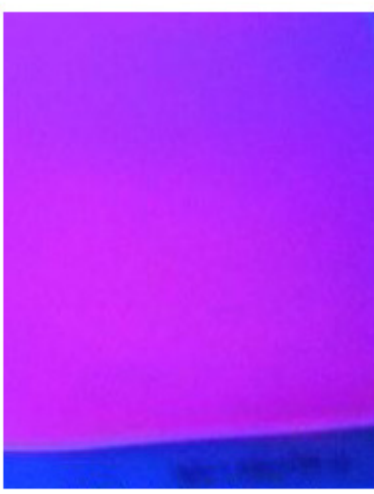

Polystyrene matrix

Figure 7. Colors of merocyanine in different polymer matrices (left) and aggregation induced fluorescence of merocyanine in polystyrene compared to the non fluorescent solution (right)

Last, not least the couple spiropyrane/merocyanine in thin films provides a textbook example for demonstrating and communicating the influence of the nano environment of photoactive molecules on their properties. Merocyanine (see Figure 3) that is generated by the irradiation of spiropyrane shows a very pronounced negative solvatochromic effect. It is visible to the naked eye in solvents (for example in the series toluene, acetone, ethanol) as well as in polymers of different polarities (see Figure 7). In environments of increasing polarities the color of the zwitterionic merocyanine changes from blue over violet to red. Actually, the related hypsochromic absorption shift results from the stabilization of the merocyanine by energetic depression of the HOMO, while the LUMO remains roughly at the same level.

Aggregation induced fluorescence can be made visible when comparing the luminescence behaviour of merocyanine in toluene solution and in polystyrene matrix (Figure 7). While merocyanine in toluene solution doesn't fluoresce, immobilized in polystyrene it shows a considerable reddish fluorescence [11]. This is caused by the restriction of intramolecular motions (vibrations and rotations) in the matrix compared to the situation in solution and is in good agreement with literature data [12].

\section{References}

[1] http://www.chemiedidaktik.uni-wuppertal.de/english/index.html
[2] N. J. Turro, Modern Molecular Photochemistry, Benjamin/Cummings, New York, 1978.

[3] M. W. Tausch, S. Korn: "A Laboratory Simulation for Coupled Cycles of Photosynthesis and Respiration" in Journal of Chemical Education, 78 (9), 1238 (2001).

[4] M. W. Tausch, C. Bohrmann: "Photogalvanic Cells for Classroom Investigations - A Contribution for the Ongoing Curriculum Modernization" bei Journal of Chemical Education, 80 (12), 1471 (2003).

[5] M. W. Tausch, C. Bohrmann-Linde, J. Ibanez, D.Zavala-Araiza, B Sotomayor-Martinez Barranco, J. Torres-Perez, C. CamachoZuniga: "A Demonstration of Simultanous Electrochemoluminescence", Journal of Chemical Education, 90 (4), 470 (2013).

[6] M. W. Tausch, A. Banerji, U. Scherf: "Classroom Experiments and Teaching Materials on OLEDs with Semiconducting Polymers", Education Quimica, 24 (1), 17 (2013).

[7] http://www.chemiedidaktik.uni-wuppertal.de/lehre/photomol/index.html

[8] E. D. Bergmann, A. Weizmann, E. Fischer, JACS, 72, 5009 (1950).

[9] M. W. Tausch: "Teaching Photochemistry with Microscale Experiments", Education Quimica, 16 (4), 529 (2005).

[10] M. W. Tausch, S. Spinnen, M. Essers, S. Krees: „Light Absorption and Emission in Solutions and Solid Matrices", Praxis der Naturwsseschaften-Chemie in der Schule, 63, (2) 35 (2014) (German).

[11] M. W. Tausch, S. Spinnen: "A Multiple Chameleon Photochromism, Solvatochromism and Aggrgation Induced Fluorescence", Praxis der Naturwsseschaften-Chemie in der Schule, 64 (6), 46 (2015) (German).

[12] J. Mei, Y. Hong, J. W. Y. Lam, A. Qin, Y. Tang, B. Zhong Tang: "Aggregation-Induced Emission: The Whole Is More Brilliant than the Parts", Advanced Materials, 26 (31), 5429 (2014). 